\title{
Dolor torácico
}

\author{
D. Crespo Marcos, FJ. Pérez Lescure-Picarzo, M. Zambrano Castaño \\ Pediatras. Cardiología Infantil. Unidad de Pediatría. \\ Hospital Universitario Fundación Alcorcón. Alcorcón, Madrid. España.
}

\section{Resumen}

El dolor torácico es un motivo de consulta relativamente poco frecuente en niños y adolescentes. No obstante, genera gran ansiedad y preocupación al niño y a su familia, pues es percibido con frecuencia como una patología cardíaca potencialmente letal. Presentamos una revisión del tema, incidiendo sobre el diagnóstico y el manejo inicial de estos pacientes.

Palabras clave: Dolor torácico. Patología cardíaca. Manejo inicial.

\section{Chest pain}

\section{Abstract}

Chest pain is a relatively uncommon reason for consultation during childhood and adolescence. Nevertheless, it may be alarming for the patient and family, due to the fact that it is usually misinterpreted as a potentially lethal cardiac disease. We present a review about this topic, focusing on diagnosis and initial management of these patients.

Key words: Chest pain. Cardiac disease. Initial management.

\section{Introducción}

El dolor torácico supone el 0,25-0,5\% de las consultas en los servicios de urgencias pediátricos (1 de cada 200-400 niños $)^{1-3}$. Genera gran ansiedad y preocupación al niño y a su familia pues es, con frecuencia, percibido como una patología cardíaca potencialmente letal. Si bien presenta un origen cardiovascular en muy pocos casos (0-4\% $)^{1-3}$, debemos tenerlo presente en el diagnóstico diferencial.

Casi siempre se trata de un proceso benigno, aunque muchas veces es recurrente, prolongado y difícil de tratar. No tiene preferencia por un sexo y predomina en adolescentes. Cuando se da en menores de 11 años, la probabilidad de una base orgánica subyacente es superior $^{2}$.

David Crespo Marcos, dcrespo@fhalcorcon.es

Los autores declaran no presentar conflictos de intereses en relación con la preparación y publicación de este artículo. 


\section{Etiología}

Las principales causas ${ }^{2,3}$ se enumeran en la tabla 1.

\section{Clínica}

Las manifestaciones clínicas dependen de la causa subyacente ${ }^{2,3}$ :

1. Idiopática (12-85\%): episodios intensos, cortos y recurrentes, sin otros síntomas asociados, relacionados o no con el ejercicio. Puede producir una ansiedad importante y llegar a interferir con las actividades cotidianas, aunque no es lo habitual. No suele tener una evolución prolongada (dura unas semanas o unos pocos meses). La exploración física es normal y el dolor no es reproducible.

\begin{tabular}{|c|c|}
\hline \multicolumn{2}{|l|}{ 1. Idiopático (12-85\%) } \\
\hline 2. Musculoesquelético (15-43\%) & $\begin{array}{l}\text { - Traumatismo } \\
\text { - Sobrecarga muscular } \\
\text { - Costocondritis } \\
\text { - Síndrome de Tietze } \\
\text { - Síndrome de pinzamiento precordial } \\
\text { - Síndrome de la costilla deslizante }\end{array}$ \\
\hline 3. Respiratorio $(12-21 \%)$ & $\begin{array}{l}\text { - Tos, asma (inducido por el ejercicio) } \\
\text { - Neumonía } \\
\text { - Derrame pleural } \\
\text { - Neumotórax } \\
\text { - Neumomediastino } \\
\text { - Pleurodinia }\end{array}$ \\
\hline 4. Psicógeno (5-17\%) & $\begin{array}{l}\text { - Hiperventilación } \\
\text { - Síntomas de conversión }\end{array}$ \\
\hline 5. Gastrointestinal (4-7\%) & $\begin{array}{l}\text { - Reflujo gastroesofágico } \\
\text { - Esofagitis } \\
\text { - Gastritis } \\
\text { - Ingesta de cuerpo extraño/cáustico } \\
\text { - Colecistitis }\end{array}$ \\
\hline 6. Cardiovascular (0-4\%) & $\begin{array}{l}\text { - Anomalías estructurales (cardiopatías con obstrucción del } \\
\text { TSVI/prolapso de la válvula mitral/arterias coronarias anómalas) } \\
\text { - Arritmias (taquiarritmias, extrasistolia) } \\
\text { - Fenómenos inflamatorios (miocarditis, pericarditis) } \\
\text { - Enfermedad de Kawasaki } \\
\text { - Vasoespasmo, oclusión de las arterias coronarias } \\
\text { - Rotura o disección aórtica/TEP }\end{array}$ \\
\hline 7. Otros $(4-21 \%)$ & $\begin{array}{l}\text { - Mastalgia } \\
\text { - Herpes zóster }\end{array}$ \\
\hline
\end{tabular}


2. Musculoesquelética (15-43\%): suele existir antecedente de traumatismo o ejercicio intenso. Las molestias empeoran con los movimientos y con la respiración profunda.

a) Costocondritis (9-22\%): episodios de dolor leve-moderado en la cara anterior del tórax, en las uniones condrocostal, esternoclavicular o esternocostal. Puede afectar a uno o más cartílagos costales. Suele ser unilateral aunque también puede ser bilateral. Lo más frecuente es que se produzca en la 4. ${ }^{\mathrm{a}}-6$. $^{\mathrm{a}}$ unión condrocostal izquierda. Es reproducible a la palpación, puede persistir varios meses y es más frecuente en niñas.

b) Síndrome de Tietze: rara variante de la costocondritis. Afecta a los cartílagos costales superiores (habitualmente a la 2. ${ }^{a}$ unión costocondral o esternoclavicular derecha) y cursa con tumefacción evidente (a veces con eritema). Puede prolongarse durante meses o años.

c) Síndrome de pinzamiento precordial (o punzada de Teixidor)" es un dolor intenso de inicio brusco y duración breve (segundos o minutos) localizado en el borde esternal izquierdo. Ocurre en reposo o tras ejercicio poco intenso, muchas ve- ces al flexionar el tronco o al sentarse con los hombros caídos. Empeora con la respiración profunda y mejora en sedestación con la espalda bien erguida. Tiene carácter recurrente, su frecuencia y duración son muy variables, y no es reproducible.

d) Síndrome de la costilla deslizante 5 : dolor en relación con las costillas 8. ${ }^{a}-10{ }^{a}$, que no están fijadas directamente al esternón y se encuentran unidas entre sí mediante tejido fibroso. Por laxitud de éste, pueden deslizarse una sobre otra y provocar un chasquido seguido de un dolor intenso en el borde costal inferior. Se suele relacionar con el ejercicio físico, y el dolor puede persistir durante horas. Es reproducible traccionando con los dedos el borde costal hacia delante, mediante flexión del tronco o con inspiración profunda.

3. Respiratoria (12-21\%):

a) Asma/tos: son las principales causas respiratorias y generalmente están relacionadas con la sobrecarga muscular. No es infrecuente que sean remitidos a cardiología niños con asma inducido por el ejercicio, pues refieren dolor torácico opresivo y disnea en relación 
con la actividad física. En tales casos conviene indagar sobre la presencia de tos o ruidos respiratorios, así como saber que muchas veces únicamente se quejan de escasa resistencia al ejercicio.

b) Neumonía: se suele asociar a dolor torácico (en ocasiones es referido como abdominal), fiebre y otros síntomas respiratorios.

c) Derrame pleural: dolor que empeora con movimientos respiratorios profundos. Puede asociarse a fiebre o disnea. Pueden objetivarse matidez en la percusión torácica e hipoventilación.

d) Neumotórax: dolor torácico brusco e intenso que se irradia a la espalda, acompañado de disnea. Puede haber antecedente traumático. Es más frecuente en personas con fenotipo leptosómico (altas y delgadas). En la auscultación pulmonar se puede objetivar hipoventilación.

e) Neumomediastino: inicio brusco con dolor torácico, disnea y disfagia. Es más frecuente con fenotipo leptosómico y tras la inhalación de drogas vasoconstrictoras (cocaína, marihuana o éxtasis). En la auscultación cardíaca se suele identificar una crepitación sincrónica con la sístole (signo de Hamman). Puede haber enfisema subcutáneo.

f) Pleurodinia: muy poco frecuente. Se produce cuatro días después de la infección por enterovirus y presenta inicio brusco de fiebre y dolor torácico (intenso, espasmódico, dura minutos $u$ horas, presenta cortejo vegetativo y afectación del estado general y empeora con la respiración). La auscultación pulmonar y la radiografía de tórax son normales.

4. Psicógena $(5-17 \%)^{6}$ : suele tratarse de un dolor atípico, con clínica vaga, cambiante y prolongada. Se asocia con otros síntomas como cefalea, mareos, dolor abdominal y crisis de ansiedad. Casi siempre pueden identificarse acontecimientos desencadenantes (separación paterna, fallecimiento de un familiar, conflicto escolar, etc.). Es más frecuente en mujeres adolescentes.

5. Gastrointestinal (4-7\%):

a) Reflujo gastroesofágico ${ }^{7}$ : es la causa digestiva más frecuente. Es un dolor retroesternal quemante ascendente tras las comidas que empeora en decúbito.

b) Esofagitis: dolor retroesternal intenso en relación con la ingesta. Puede acompañarse de disfagia. A veces puede objetivarse dolor en 
la palpación epigástrica, en posible relación con una gastritis subyacente.

c) Ingesta de cuerpo extraño/cáustico: debe sospecharse en niños pequeños. Dolor/molestias a nivel retroesternal. Puede acompañarse de disfagia y sialorrea, de etiología no aclarada.

d) Colecistitis: dolor pospandrial tipo cólico en el hipocondrio derecho.

6. Cardiovasculares (0-4\%):

a) Arritmias ${ }^{2,3}$ :

- Taquiarritmias: la taquicardia supraventricular es percibida con frecuencia como un dolor torácico, sobre todo en niños pequeños. Los episodios suelen tener inicio y fin bruscos y asocian fatiga y cortejo vegetativo. Pueden estar relacionados o no con el ejercicio físico. El electrocardiograma (ECG) basal puede ser normal (lo más frecuente) o mostrar anomalías que predispongan a padecer taquiarritmias (QT largo, QT corto, síndrome de Brugada, displasia arritmogénica del ventrículo derecho, síndromes de preexcitación).

- Extrasistolia: en ocasiones, los niños pueden referir los latidos ectópicos prematuros (supraventri- culares o ventriculares) como episodios breves de dolor o disconfort torácico. Muchas veces se quejan de "un vuelco al corazón" seguido de "el corazón se me para" (pausa compensatoria).

b) Anomalías estructurales ${ }^{2,3,8}$ :

- Con obstrucción del tracto de salida ventricular izquierdo: las más frecuentes son la estenosis aórtica (subvalvular, valvular o supravalvular) y la miocardiopatía hipertrófica obstructiva. Pueden producir síncope o muerte súbita, así como dolor torácico de tipo isquémico (opresivo, intenso con cortejo vegetativo, precordial/retroesternal, irradiado al cuello, a uno o ambos brazos, a la espalda o al abdomen, en el contexto de estrés físico o psíquico, o tras una comida copiosa). En la auscultación cardíaca suele llamar la atención un soplo sistólico claramente audible, con máxima intensidad en el borde esternal derecho alto, que aumenta en bipedestación y mediante maniobra de Valsalva. En el ECG se suelen objetivar signos de hipertrofia ventricular izquierda u ondas $Q$ patológicas.

- Prolapso de la válvula mitral: 
asociación muy controvertida ${ }^{9}$ entre esta anomalía valvular y el dolor torácico (localizado en ápex, de corta duración, no relacionado con ejercicio ni con estrés emocional; atribuido a isquemia endomiocárdica del ventrículo izquierdo o de los músculos papilares). Hallazgos frecuentes en pacientes con prolapso mitral: chasquido mesosistólico seguido de soplo telesistólico (sin insuficiencia) e inversión de la onda $T$ en derivaciones de cara inferior (II, III, aVF). El prolapso mitral es muy frecuente en caso de conectivopatía (Marfan, Ehlers-Danlos).

- Arterias coronarias anómalas: en general producen una repercusión hemodinámica importante en el período de lactante, aunque posteriormente se pueden manifestar como muerte súbita o síntomas relacionados con el ejercicio (síncope, dolor torácico de tipo isquémico). El ECG puede mostrar signos de isquemia miocárdica o de infarto antiguo, si bien puede ser completamente normal.

c) Fenómenos inflamatorios ${ }^{2,3,8,10}$ : - Miocarditis: los niños mayores pueden quejarse de dolor torácico. Siempre se acompaña de taquicardia y suele asociarse a palpitaciones, disnea de esfuerzo y afectación del estado general. En la auscultación cardíaca se pueden objetivar tonos apagados $y$ ritmo de galope. El ECG muestra potenciales disminuidos, depresión del segmento ST e inversión de la onda $T$.

- Pericarditis: dolor precordial/retroesternal de instauración relativamente rápida aunque no brusca, intenso (no opresivo, sin cortejo vegetativo), que aumenta con movimientos torácicos, inspiración, decúbito y tos, y mejora en sedestación con el tronco inclinado hacia delante. Puede auscultarse un roce pericárdico. En el ECG se objetiva una elevación difusa del segmento ST con o sin un descenso del segmento PR.

d) Enfermedad de Kawasaki: ante niños que la hayan padecido debemos considerar firmemente la posibilidad de un origen isquémico coronario ante dolor torácico intenso. Tras la fase inflamatoria vasculítica inicial, se puede producir (sobre todo si se dan aneurismas corona- 
rios) reemplazo por tejido fibroso, causando estenosis en las arterias coronarias.

e) Vasoespasmo coronario/vasooclusión coronaria:

- Vasoespasmo coronario primario: extremadamente infrecuente, pero bien descrito en la literatura pediátrica. Dolor torácico de tipo isquémico. El ECG muestra la elevación del segmento ST y la inversión de la onda T. En la analítica sanguínea se elevan los enzimas cardíacos (CPK-MB y troponinas). Puede llegar a ocasionar infarto.

- Vasoespasmo coronario secundario al consumo de cocaína: el niño presenta los mismos hallazgos que en el vasoespasmo primario. Se encuentra ansioso/confuso y frecuentemente hipertenso.

- Vasooclusión coronaria: puede darse en niños con drepanocitosis. Es muy importante en estos pacientes asegurar un adecuado estado de hidratación para evitar el desarrollo de crisis vasooclusivas en las arterias coronarias o en otros territorios.

f) Vasculares:

- Rotura o disección aórtica: muy infrecuente, aunque existe riesgo aumentado en conectivopatías (sobre todo en el síndrome de Marfan), síndrome de Turner, síndrome de Noonan y pacientes con válvula aorta bicúspide. Produce dolor torácico/abdominal muy intenso, disnea y signos de bajo gasto.

- Tromboembolismo pulmonar ${ }^{10}$. inicio brusco con dolor pleurítico, disnea, tos, taquicardia y taquipnea. En ocasiones asocia fiebre y hemoptisis. Factores de riesgo: toma de anticonceptivos orales, traumatismos graves con fracturas, inmovilidad prolongada, cardiopatías congénitas con cortocircuitos intracardíacos, obesidad mórbida y derivación ventriculoatrial por hidrocefalia. Se puede orientar el diagnóstico mediante analítica sanguínea (elevación del dímero D), ECG (signos de sobrecarga derecha, patrón S1Q3T3: onda $S$ en I, onda $Q$ en III, inversión de la onda T en III), ecocardiografía (dilatación del ventrículo derecho, aumento de las presiones vasculares pulmonares) y radiografía de tórax (hiperclaridad segmentaria, atelectasia basal laminar, derrame pleural, infiltrado alveolar triangular). Se confirma mediante tomografía helicoidal o 
gammagrafía pulmonar de ventilación/perfusión.

7. Otras $(<1 \%)$ :

a) Mastalgia: se da tanto en niñas como en niños. En niñas pospuberales suele deberse a lesiones quísticas. En edad puberal generalmente forma parte en ambos sexos del desarrollo normal.

b) Herpes zoster: dolor muy intenso, unilateral, localizado en un dermatoma. Hipersensibilidad de la zona afecta. Precede en unos días a la erupción vesicular.

\section{Aproximación diagnóstica}

El objetivo principal es descartar una causa cardiovascular del dolor, que es, además, el principal motivo de preocupación en el paciente y la familia ${ }^{2,3,8,11,12}$.

\section{Anamnesis}

a) Características del dolor:

- Inicio y fin: cuando son bruscos y acompañados de palpitaciones orienta hacia las taquicardias paroxísticas.

- Intensidad del dolor: dolores muy intensos orientan hacia una patología orgánica en general y cardíaca en particular. Hay que tener cuidado si interfieren con las actividades cotidianas o asocian cortejo vegetativo.
- Duración y periodicidad: las dolencias de larga evolución tienen menor probabilidad de deberse a una base orgánica.

- Tipo de dolor: el dolor isquémico es opresivo, el musculoesquelético y el idiopático se definen muchas veces como tipo pinchazo, el retroesternal quemante orienta hacia reflujo gastroesofágico/esofagitis.

- Localización e irradiación: en general, un dolor localizado y que no irradia es menos sugestivo de enfermedad grave.

- Condicionantes: la relación con la ingesta orienta hacia una patología digestiva. Cuando la clínica se da única o preferentemente durante el ejercicio físico, hay que sospechar patología cardíaca/asma inducidos por el ejercicio. Se debe indagar sobre posibles traumatismos o ejercicio intenso (musculoesquelético), tos intensa o persistente (respiratorio), ansiedad o ingesta de drogas (cocaína). Un dolor nocturno que despierta al niño orienta a organicidad.

- Relación con postura/respiración/ movimientos: el reflujo gastroesofágico empeora en decúbito. La pericarditis empeora en decúbito y 
mejora en sedestación inclinando el tronco hacia delante. Un empeoramiento con la respiración profunda sugiere irritación pleural o patología de la pared torácica. El empeoramiento con los movimientos orienta hacia una causa musculoesquelética.

- Síntomas acompañantes: su presencia hace que sea más probable la existencia de un sustrato orgánico. Fiebre y disnea orientan hacia la neumonía; síncope y cortejo vegetativo hacia la cardiopatía; palpitaciones hacia la taquiarritmia; derrame pleural y síndrome general, en ausencia de neumonía, pueden orientar hacia un proceso neoplásico.

b) Antecedentes:

- Personales: indagar sobre la presencia de enfermedades de base que puedan orientar hacia el origen del dolor: asma; cardiopatía congénita no corregida/corregida pero con lesiones residuales importantes; válvula aorta bicúspide (rotura/disección aórtica); miocardiopatía; taquiarritmias patológicas o sustrato arritmogénico como QT largo, QT corto, síndromes de preexcitación o síndrome de Brugada; trastornos de la coagulación (embolismo pulmonar); conectivopatía (prolapso mitral, disección aórtica); determinados síndromes como Noonan o Turner (rotura/disección aórtica); enfermedad de Kawasaki (isquemia coronaria); y drepanocitosis (vasooclusión coronaria).

- Familiares: interrogar acerca de la muerte súbita de origen cardíaco o desconocido, especialmente en personas menores de 50 años; miocardiopatías, sobre todo hipertrófica; taquiarritmias o sustrato arritmogénico como QT largo, QT corto, síndromes de preexcitación o Brugada.

\section{Exploración física}

Antes de centrarnos en el tórax, se deben tomar las constantes del paciente (frecuencia cardíaca, frecuencia respiratoria, saturación transcutánea de oxígeno y tensión arterial) y realizar una exploración física general. La existencia de afectación del estado general orienta hacia una patología grave, aunque en muchas ocasiones es secundaria a la gran ansiedad y preocupación que este proceso conlleva.

- Abdomen: debe valorarse cuidadosamente, pues no es infrecuente que los niños refieran dolor torácico ante trastornos abdominales. 
- Tórax: se debe inspeccionar y palpar la totalidad del tórax (también las regiones mamarias), incluyendo cada unión condrocostal y condroesternal (para ello emplearemos el pulpejo de los dedos), así como los principales grupos musculares (pectorales, hombros, dorsales, lumbares). La reproducción del dolor con estas maniobras es diagnóstica de origen musculoesquelético (no se precisará por lo general de la realización de pruebas complementarias).

- Auscultación cardíaca: evaluar la presencia de soplos patológicos (cardiopatías congénitas/miocardiopatía hipertrófica), roces (pericarditis), tonos apagados (miocarditis, derrame pericárdico) o crepitación sincrónica con la sístole (neumomediastino).

- Auscultación pulmonar: evaluar la ventilación en todos los segmentos pulmonares (hipoventilación en neumotórax, neumonía, derrame pleural), soplo tubárico (derrame pleural) y estertores (neumonía).

\section{Pruebas complementarias}

No siempre deben solicitarse ante un niño con dolor torácico (por ejemplo, si no es un dolor muy intenso y se ha llegado a un diagnóstico tras la anamnesis y la exploración física). En ocasiones están indicadas en casos de gran ansiedad tanto en el niño como en su familia, pues su realización tiene un efecto tranquilizante, si bien conviene tener en cuenta que a veces les refuerza la idea de que padece un problema orgánico grave.

a) Radiografía de tórax:

- Indicaciones: dolor muy intenso, sospecha de fractura costal, traumatismo potencialmente grave (accidente de tráfico, caída de altura), sospecha de ingesta de cuerpo extraño (o un cáustico) y gran ansiedad en el niño o la familia.

- Evaluar: las estructuras óseas, el parénquima y la vascularización pulmonar, la presencia de aire ectópico, la silueta cardíaca y de los grandes vasos.

b) ECG ${ }^{13-15}$ :

- Indicaciones: dolor muy intenso; dolor desencadenado con el ejercicio; asociado a síncope, palpitaciones o taquicardia; datos sugestivos de dolor isquémico; gran ansiedad en el niño o la familia.

- Evaluar: ondas Q patológicas (miocardiopatía hipertrófica, infar- 
to antiguo); signos de hipertrofia ventricular izquierda grave (miocardiopatía hipertrófica, cardiopatía congénita con obstrucción al tracto de salida ventricular izquierdo); elevación del segmento ST (pericarditis, isquemia); inversión de la onda T (miocardiopatía, hipertrofia ventricular grave); sustrato arritmogénico (síndromes de preexcitación, QT largo, QT corto, síndrome de Brugada).

c) Otras:

- Analítica sanguínea: valorar hemograma y rectantes de fase aguda en procesos infecciosos/inflamatorios (neumonía, miocarditis, pericarditis); enzimas cardíacos (CPK-MB, troponinas) en sospecha de isquemia coronaria/miocarditis/pericarditis.

- Otras pruebas de imagen: se valorarán de forma individualizada en función de la situación clínica y la sospecha diagnóstica concreta: ecocardiografía (cardiopatía congénita, miocardiopatía, tromboembolismo pulmonar, miocarditis, pericarditis), tomografía computarizada (politraumatismo; helicoidal si se sospecha de tromboembolismo pulmonar), resonancia magnética, etc.

\section{Tratamiento}

Será individualizado según la etiología $a^{2,3,6,8,11}$. Existe una pauta general orientativa:

- Idiopático o musculoesquelético: tranquilizar al niño y a la familia, insistiendo acerca de la benignidad del proceso. Valorar los antiinflamatorios y el reposo relativo.

- Respiratorio: tratamiento de asma (tos, neumonía) según los protocolos específicos. Drenar el neumotórax si se produce repercusión. En neumomediastino, generalmente hay que mantener una actitud conservadora (analgesia, reposo relativo).

- Digestivo: ante un reflujo gastroesofágico o esofagitis, se debe pautar un tratamiento antiácido y remitir a la consulta de digestivo. La actuación se llevará a cabo según protocolos en ingesta de cáustico o cuerpo extraño.

- Psicógeno: valorar la asistencia psiquiátrica.

- Cardíaco: en función de la causa.

\section{Criterios de derivación}

Se detallan en el algoritmo de manejo inicial de la figura 1. Se derivarán al hospital aquellos casos en que esté indicado el ingreso, o bien ante la necesi- 


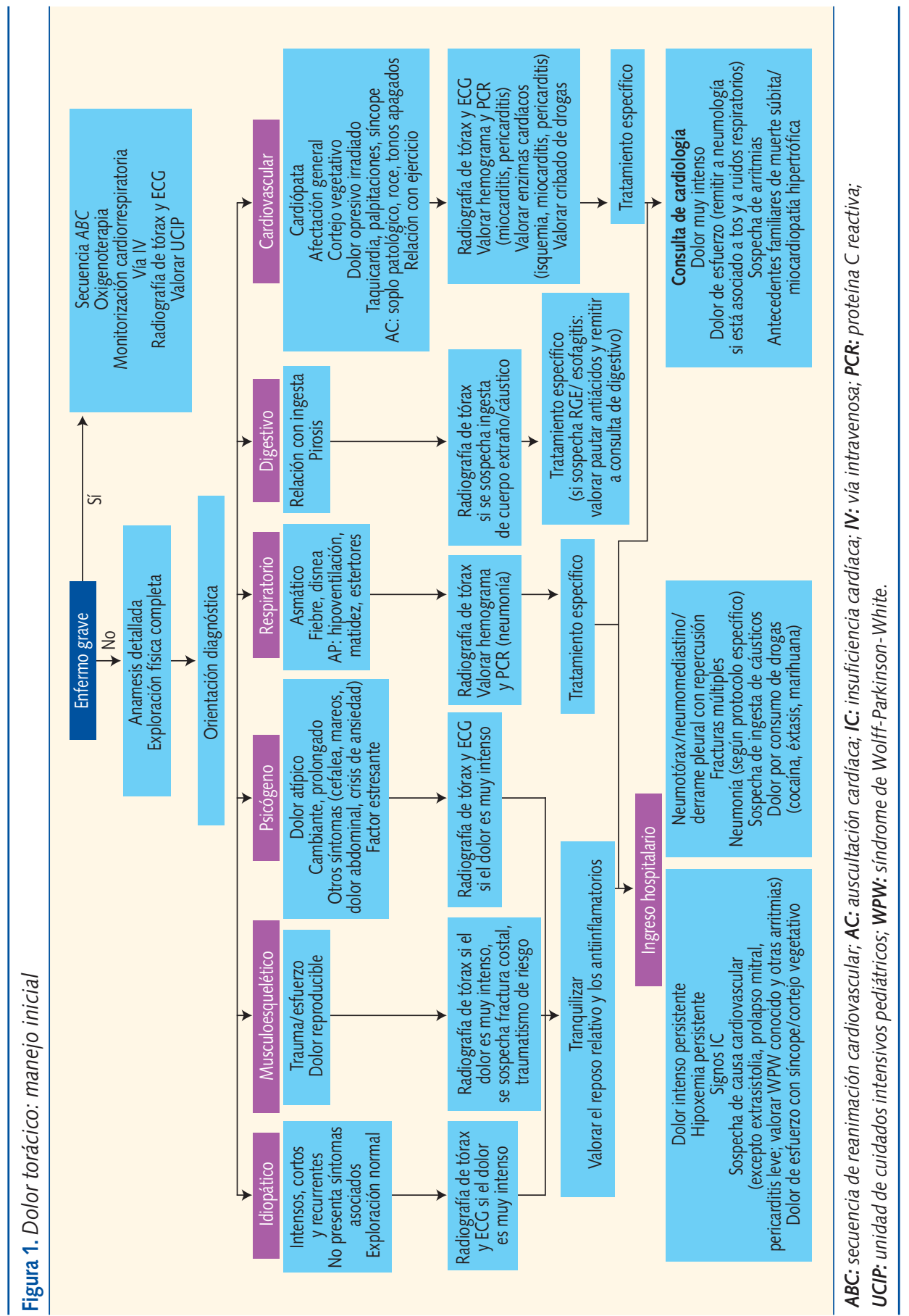


dad de realizar pruebas complementarias que no estén disponibles en Atención Primaria. Se enumerarán los crite- rios por los que el niño debe ser remitido a la consulta ordinaria de cardiología pediátrica.

\section{Bibliografía}

1. Gastesi Larrañaga $M$, Fernández Landaluce A, Mintegui Raso S, Vázquez Ronco M, Benito Fernández J. Dolor torácico en urgencias de pediatría: un proceso habitualmente benigno. An Pediatr (Barc). 2003;59:234-8.

2. Cava JR, Sayger PL. Chest pain in children and adolescents. Pediatr Clin N Am. 2004;51:55368.

3. Park MK. Child with chest pain. In: Park MK, editor. Pediatric Cardiology for practitioners. 5. ${ }^{a}$ ed. Philadelphia: Mosby, Elsevier Health Science; 2008. p. 499-507.

4. Gumbiner $\mathrm{CH}$. Precordial catch syndrome. South Med J. 2003;96:38-41.

5. Porter GE. Slipping rib syndrome: an infrequently recognized entity in children: a report of three cases and review of the literature. Pediatrics. 1985;76:810-3.

6. Anzai AK, Merkin TE. Adolescent chest pain. Am Fam Physician. 1996;53:1682-8.

7. Richter JE. Gastroesophageal reflux disease as a cause of chest pain. Medical Clin N Am. 1991; 75:1065-80.

8. García Angleu A, González Vila L, Herrera del Rey C. Dolor torácico en el niño. Protocolos de la Sociedad Española de Cardiología Pediátrica, 2005 [consultado el 11/11/2009]. Disponible en www. secardioped.org/protocolos/protocolos/Capitulo _27.pdf
9. Alpert MA, Mukerji V, Sabeti M, Russell $\mathrm{JL}$, Beitman BD. Mitral valve prolapse, panic disorder, and chest pain. Med Clin N Am. 1991; 75:1119-33.

10. Bonillo Perales A, Batlles Garrido J, Rubí Ruiz T, González Jiménez Y, Aguirre Rodríguez J, Muñoz Hoyos A. Tromboembolismo pulmonar. An Pediatr (Barc). 2003;58:22-9.

11. Ferrés i Serrat F, García Algas F. Dolor torácico. Protocolos de Urgencias de la Asociación Española de Pediatría, 2008 [consultado el 11/11/ 2009]. Disponible en www.aeped.es/protocolos/ urgencias/9.pdf

12. Benito FJ, Mintegui S, Sánchez J. Dolor torácico. Diagnóstico y tratamiento de urgencias pediátricas. 2009. Hospital de Cruces [consultado el 11/11/2009]. Disponible en http://urgenciaspe diatria.hospitalcruces.com/doc/generales/proto/ Cap10.2dolortoracico.pdf

13. Pérez-Lescure Picarzo FJ. Guía rápida para la lectura sistemática del ECG pediátrico. Rev Pediatr Aten Primaria. 2006;8:319-26.

14. Pérez-Lescure FJ, Echávarri Olavarría F. El electrocardiograma en Pediatría de Atención Primaria (I). Aspectos generales, indicaciones e interpretación sistemática. Rev Pediatr Aten Primaria. 2005;7:277-302.

15. Pérez-Lescure FJ, Echávarri Olavarría F. El electrocardiograma en Pediatría de Atención Primaria (II). Cambios relacionados con la edad y arritmias básicas. Rev Pediatr Aten Primaria. 2005; 7:463-80. 